Journal of Animal and Veterinary Advances 10 (13): 1694-1698, 2011

ISSN: $1680-5593$

(C) Medwell Journals, 2011

\title{
The Analysis of ERIC-PCR Genomic Polymorphism of Salmonella Isolated Strains in Pig Carcass
}

\author{
Li Yu, Fang Yan-Hong, Zhu Heng-Wen, Sun Pei, \\ Wei Jian-Zhong and Yin Zhong-Jun \\ College of Animal Sciences and Technology, Anhui Agricultural University, 230036 Hefei, China
}

\begin{abstract}
To investigate molecular epidemiological characteristic of Salmonella isolated strains in pig carcass, the current study, 71 strains of Salmonella genome DNA were isolated from the muscle and surface of pig carcass samples in five designated slaughterhouses in Hefei, Anhui province, China. They were gathered and analyzed using the technique of Enterobacterial Repetitive Intergenic Consensus-Polymerase Chain Reaction (ERIC-PCR). The multiple DNA fragments of all strains generated with ERIC2 primers were composed of 6-14 bands ranging in sizes between $250 \mathrm{bp}$ and $3 \mathrm{~kb}$. One characteristic band (approximately $250 \mathrm{bp}$ ) was conserved among nearly all isolates. This study showed that genotype polymorphism exists in Salmonella isolated strains, serotype and the source of the isolated strains with the same genotype had certain relevance. It further proves that the carcass surface contamination during slaughter is mainly from cross-contamination and environmental pollution during product processing and carcass meat-like isolates were from the endogenous contamination.
\end{abstract}

Key words: Salmonella, pig carcass, ERIC-PCR, genotyping, spollution, China

\section{INTRODUCTION}

Salmonella is an important cause of food-borne infections with a broad host spectrum. Pigs are one of the main reservoir hosts. To date, $>2,500$ Salmonella serovars have been identified. Traditional serological methods have some limitations such that some strains cannot be identified serologically (Rasschaert et al., 2005). The deficiencies of the serological typing are due to several reasons: Salmonella antigens can have mutations that include the $\mathrm{HO}, \mathrm{SR}$ variation and $\mathrm{H}$ antigen phase variation.

Also, common antigens may exist among different strains of bacteria that result in cross-reaction. Therefore to investigate the origins and the relationships among different isolates, more precise methods than serotyping are needed. Enterobacterial Repetitive Intergenic Consensus (ERIC) is found in enterobacterial genomes non-coding conserved repetitive sequences with 126 bp long.

It has species specificity in the location and number of copies of chromosome (Hulton et al., 1991; Versalovic et al., 1991). As one of main enterobacterial pathogenic bacteria, ERIC sequence exists in Salmonella genome.

The current study, 71 strains of Salmonella genome DNA were isolated from the muscle and surface of pig carcass samples in five designated slaughterhouses in Hefei, Anhui province, China. They were gathered and analyzed by using the technique of Enterobacterial Repetitive Intergenic Consensus-Polymerase Chain Reaction (ERIC-PCR). It was used to establish the DNA fingerprints among Salmonella isolated strains, perform genotyping of the Salmonella isolated strains and analyze the genetic similarity in order to provide molecular epidemiological evidence of the prevention and control of food-borne Salmonella.

\section{MATERIALS AND METHODS}

Bacterial strains: About 71 Salmonella strains were isolated from the muscle and surface of pig carcass samples in five designated slaughterhouses in Hefei, Anhui, China (coded as CR, SLH, HS, ZT, WW). The experiment was conducted in Animal Infectious Disease Laboratory of Anhui Agricultural University. Table 1 shows the information of isolated strains (Yu et al., 2007).

Main reagents: TSB broth is provided by Hangzhou Microbial Reagent Co., Ltd. Bacterial genomic DNA extraction kit was purchased from Tiangen Biotech (Beijing) Co., Ltd. PCR-related reagents $(10 \times \mathrm{Ex}$ Taq buffer (with $\mathrm{Mg}^{2+}$ ), $2.5 \mathrm{mM}$ dNTP, $5 \mathrm{U} \mathrm{uL}^{-1}$ Ex Taq DNA polymerase, $6 \times$ loading buffer) were purchased from

Corresponding Author: Wei Jian-Zhong, College of Animal Sciences and Technology, Anhui Agricultural University, 230036 Hefei, China 
Table 1: List of the ERIC-PCR genotypes and related information for the 71 Salmonella strains

\begin{tabular}{|c|c|c|c|c|c|c|c|c|c|}
\hline Strain No. & Genotypes & Serotypes & Isolate source & Site of origin & Strain No. & Genotypes & Serotypes & Isolate source & Site of origin \\
\hline 1 & I & S. reading & Surface & CR & 37 & $X V$ & S. derby & Surface & WW \\
\hline 2 & I & S. reading & Surface & $\mathrm{CR}$ & 38 & $X V$ & S. derby & Surface & WW \\
\hline 3 & I & S. reading & Surface & CR & 39 & $X V$ & S. derby & Surface & HS \\
\hline 4 & I & S. reading & Surface & $\mathrm{CR}$ & 40 & $\mathrm{XV}$ & S. derby & Muscle & HS \\
\hline 5 & I & S. reading & Surface & $\mathrm{CR}$ & 41 & $\mathrm{XV}$ & S. senfienberg & Surface & WW \\
\hline 6 & I & S. reading & Surface & $\mathrm{CR}$ & 42 & $\mathrm{XV}$ & S. senfienberg & Surface & WW \\
\hline 7 & I & S. reading & Surface & $\mathrm{CR}$ & 43 & $\mathrm{XV}$ & S. senfienberg & Surface & Ww \\
\hline 8 & I & S. reading & Surface & CR & 44 & $\mathrm{x}$ & S. senfienberg & Surface & WW \\
\hline 9 & II & S. reading & Surface & $\mathrm{CR}$ & 45 & XVII & S. senftenberg & Surface & SLH \\
\hline 10 & IV & S. reading & Muscle & $\mathrm{CR}$ & 46 & XIV & S. senffenberg & Surface & SLH \\
\hline 11 & I & S. reading & Muscle & $\mathrm{CR}$ & 47 & VII & S. senfienberg & Surface & SLH \\
\hline 12 & II & S. reading & Muscle & $\mathrm{CR}$ & 48 & XVIII & S. senfienberg & Surface & $\mathrm{CR}$ \\
\hline 13 & III & S. reading & Muscle & CR & 49 & I & S. sainpaul & Muscle & HS \\
\hline 14 & IV & S. reading & Muscle & $\mathrm{CR}$ & 50 & I & S. sainpaul & Muscle & HS \\
\hline 15 & $\mathrm{IV}$ & S. reading & Murface & WW & 51 & $\mathrm{~V}$ & S. sinstorf & Muscle & $\mathrm{CR}$ \\
\hline 16 & VI & S. reading & Surface & WW & 52 & $\mathrm{~V}$ & S. sinstorf & Surface & $\mathrm{ZT}$ \\
\hline 17 & VII & S. reading & Surface & Ww & 53 & V & S. sinstorf & Muscle & HS \\
\hline 18 & V & S. reading & Surface & $\mathrm{ZT}$ & 54 & V & S. ughelli & Muscle & CR \\
\hline 19 & V & S. muenster & Surface & ZT & 55 & V & S. ughelli & Surface & SLH \\
\hline 20 & V & S. muenster & Surface & ZT & 56 & V & S. ughelli & Surface & $\mathrm{ZT}$ \\
\hline 21 & V & S. muenster & Surface & ZT & 57 & IV & S. nchanga & Surface & CR \\
\hline 22 & V & S. muenster & Surface & ZT & 58 & IV & S. nchanga & Surface & CR \\
\hline 23 & V & S. muenster & Surface & ZT & 59 & VI & S. nchanga & Surface & $\mathrm{ZT}$ \\
\hline 24 & V & S. muenster & Surface & HS & 60 & XVI & S. nchanga & Surface & HS \\
\hline 25 & V & S. muenster & Muscle & HS & 61 & XVI & S. nchanga & Muscle & HS \\
\hline 26 & XI & S. muenster & Muscle & HS & 62 & V & S. vejle & Surface & $\mathrm{ZT}$ \\
\hline 27 & V & S. muenster & Muscle & SLH & 63 & VI & S. vejle & Surface & $\mathrm{ZT}$ \\
\hline 28 & V & S. muenster & Muscle & SLH & 64 & XII & S. vejle & Surface & $\mathrm{ZT}$ \\
\hline 29 & V & S. muenster & Surface & WW & 65 & VI & S. vejle & Muscle & HS \\
\hline 30 & V & S. muenster & Muscle & Ww & 66 & $\mathrm{IX}$ & S. vejle & Muscle & SLH \\
\hline 31 & V & S. muenster & Muscle & $\mathrm{CR}$ & 67 & $\mathrm{LX}$ & S. vejle & Muscle & SLH \\
\hline 32 & VIII & S. derby & Surface & SLH & 68 & $\mathrm{LX}$ & S. vejle & Muscle & SLH \\
\hline 33 & $\mathrm{XV}$ & S. derby & Surface & SLH & 69 & $\mathrm{IX}$ & S. vejle & Muscle & SLH \\
\hline 34 & VIII & S. derby & Muscle & SLH & 70 & V & S. amounderness & Muscle & HS \\
\hline 35 & VIII & S. derby & Muscle & SLH & 71 & XIII & S. amounderness & Surface & $\mathrm{ZT}$ \\
\hline$\underline{36}$ & $\mathrm{XV}$ & S. derby & Surface & Ww & - & - & - & - & - \\
\hline
\end{tabular}

Takara Co., Ltd.; DNA Marker 100-3000 bp ladder-K, primers ERIC2 (5'-AAGTAAGTGACTGGGGTGAGCG-3') (Zhang et al., 2007) was synthesize by Sangon Biotech (Shanghai) Co., Ltd.

Main equipments: Clean benchtop, incubators, high-speed refrigerated centrifuge, gradient PCR, the level of electrophoresis and TS-680D Pui Ching automatic gel image analysis instrument.

Bacterial growth: Bacteria fosters were seized $20 \mathrm{uL}$ to get inoculated in $2 \mathrm{~mL}$ TSB broth at $37^{\circ} \mathrm{C}$ oscillation shaking incubator after $6-8 \mathrm{~h}$, stored at $4^{\circ} \mathrm{C}$ for use.

Extraction of bacterial chromosomal DNA: Following the instructions of bacterial chromosomal DNA extraction kit and then place the extracted genomic DNA in $-20^{\circ} \mathrm{C}$ refrigerator for use.

ERIC-PCR: ERIC-PCR was performed with single primer ERIC2. Each of the $50 \mathrm{~mL}$ PCR volume was composed of $3 \mathrm{uL}$ template DNA; $5 \mathrm{uL} 10 \times \mathrm{Ex}$ Taq buffer (with $\mathrm{Mg}^{2+}$ ); 4 uL 2.5 mM dNTP; 4 uL 10 pM primer ERIC
2; $0.25 \mathrm{uL} 5 \mathrm{U} \mathrm{uL}^{-1} \mathrm{Ex}$ Taq DNA polymerase and with double distilled water to adjust the final volume to $50 \mathrm{uL}$. Amplification was performed in a PCR express machine by using the following program: preliminary denaturation for 5 min at $94^{\circ} \mathrm{C}, 35$ cycles of $94^{\circ} \mathrm{C}$ for $1 \mathrm{~min}, 52^{\circ} \mathrm{C}$ for $1 \mathrm{~min}$ and $72^{\circ} \mathrm{C}$ for $3 \mathrm{~min}$ followed by a final $10 \mathrm{~min}$ at $72^{\circ} \mathrm{C}$. PCR amplification products were obtained with $6 \times$ loading buffer after mixing, the $1.5 \%$ agarose gel electrophoresis $(1 \times \mathrm{TAE}$ as the electrophoresis buffer, $\mathrm{EB}$ staining, $4 \mathrm{~V} \mathrm{~cm}^{-1}$ electrophoresis under the conditions of 1.5-2 h), the DNA marker 100-3000 bp ladder-K as the molecular weight standards then electrophoresis results were detected under UV gel imaging system. All strains were repeated twice to record ERIC-PCR patterns.

Composite analysis of fingerprint and data analysis: $\mathrm{A}$ comparative analysis of electrophoresis patterns was conducted according to the analysis of fragments of the number and relative position. Clustering analysis is based on the choice of bands. ERIC-PCR products were recorded in $(0,1)$, i.e., the same moving position (the same molecular weight fragments) is marked as 1 if amplified and 0 if not. Using software, Cross-check to process to 
obtain a matrix of binary series. Data were input into the software NTSYS-pc 2.10 by using Unweighted pair group method, using averages algorithm (UPMGA) cluster analysis to plot the genetic mapping diagram. Each isolate is considered as a Taxonomic Unit (OTU) and strains with similarity $\geq 90 \%$ are consider as isolates with the same origin (Borges et al., 2003).

\section{RESULTS AND DISCUSSION}

The ERIC-PCR analyses of 71 strains of Salmonella belonging to ten different serotypes on the gels were conducted. They were all amplified with clear DNA fingerprint bands and were easy to distinguish visually (Fig. 1). The repeated experiment results confirm the repeatability of its fingerprints. The multiple DNA fragments of all strains generated with ERIC2 primers were composed of 6-14 bands ranging in sizes between $250 \mathrm{bp}$ and $3 \mathrm{~kb}$. One characteristic band (approximately $250 \mathrm{bp}$ ) was conserved among nearly all isolates (Except for 48th strain). The genetic similarity of all strains was seized $60-100 \%$ belonging to eighteen different genotypes (Table 1, I-XVIII). Where, V is the dominant genotype, accounting for $29.58 \%$ including 21 strains belonging to six different serotypes of Salmonella, involving five slaughterhouse carcass surface and muscle samples followed by the I genotype, accounting for $15.56 \%$ including 211 strains of Salmonella serotypes, involving two slaughterhouse carcass surface and muscle samples; the 3rd, XV genotype, accounting for $12.68 \%$ including nine strains belonging to Salmonella serotypes 2, 3 abattoir carcass body surface involved and muscle samples; the other genotypes in the proportion of between $1.4-7 \%$.

Recently, many studies on ERIC-PCR genotyping of Salmonella were conducted. It is possible to use the ERIC1R-ERIC2 primer set to discriminate Salmonella serotypes. All serotypes produced unique fingerprints and the isolates within one serotype had identical patterns (Van Lith and Aarts, 1994). Another study has also proved that ERIC-PCR can be used to distinguish different Salmonella serotypes (Lim et al., 2005). However, according to Burr et al. (1998) who tested the same primer set on 89 Salmonella isolates of 22 serotypes, there was no correlation between genotype and serotype (Burr et al., 1998). Rasschaert et al. (2005) applied the ERIC-PCR to the typing of eighty different serotypes and five undetermined serotype of Salmonella isolated strains. This method was also used to perform typing for 55 Salmonella strains that belonged to ten different serotypes. The conclusion showed that in certain epidemiological studies, ERIC-PCR can be used to limit the number of strains that have to be serotyped (Rasschaert et al., 2005).
In this study, the number, size and characteristic bands of the DNA fingerprints of Salmonella isolated strains obtained from the 71 pig carcass samples were consistent with other researches (Swanenburg et al., 1998; Kumar et al., 2008). The results of the genetic similarity showed that the typing results of genotype and serotype of S. sainpaul, S. sinstorf and S. ughelli were consistent. There were two different genotypes included in $S$. derby, $S$. muenster and $S$. amounderness, respectively while $S$. reading, $S$. vejle, S. senftenberg, $S$. nchanga included seven, four, six and three genotypes, respectively. It was indicated that there was no correlation between genotype and serotype. Moreover in this study, the homology of a portion of Salmonella isolated strains with different serotypes was $>90 \%$. For instance, all the S. sinstorf and S. ughelli isolated strains and most of the $S$. muenster isolated strains belonged to $\mathrm{V}$ genotype. Conversely, the homology of Salmonella isolated strains with identical serotype was $<90 \%$. For example, eighteen isolated strains of $S$. reading and eight isolated strains of $S$. senftenberg belonged to seven different genotypes and six different genotypes, respectively. This shows that different serotypes of Salmonella isolated strains can have identical origin while genotype polymorphism is shown between Salmonella isolated strains with the same genotype.

By analyzing the source of strains, some Salmonella isolated strains with identical serotype isolated from the same slaughterhouse belonged to the same genotype or the homology was higher than other strains. For example, five isolated strains of $S$. nchanga were divided into three different genotypes according to their locations and four strains of $S$. vejle isolated from SLH slaughterhouse belonged to genotype IX. This demonstrated that serotype and the source of the isolated strains with the same genotype had certain relevance. As the strain samples in this study were isolated from pig carcass in different slaughterhouses, even pig carcass in the same slaughterhouse can be from different sources. Therefore, several genotypes of Salmonella isolated strains from the same slaughterhouse with identical serotype could have diversities. For instance, fourteen strains of $S$. reading isolated from $\mathrm{CR}$ slaughterhouse belonged to genotype I-IV (1-10th represents the isolated strains of pig carcass surface samples, 11-14th represents the isolated strains of pig carcass meat samples).

From the perspective of serotypes, it was demonstrated that the carcass surface contamination during slaughter is mainly from cross-contamination and environmental pollution during product processing and carcass meat-like isolates were from the endogenous contamination (Li et al., 2008). However, based on the results above, there is no necessary correlation between serotypes and genotypes so the conclusions need to be 

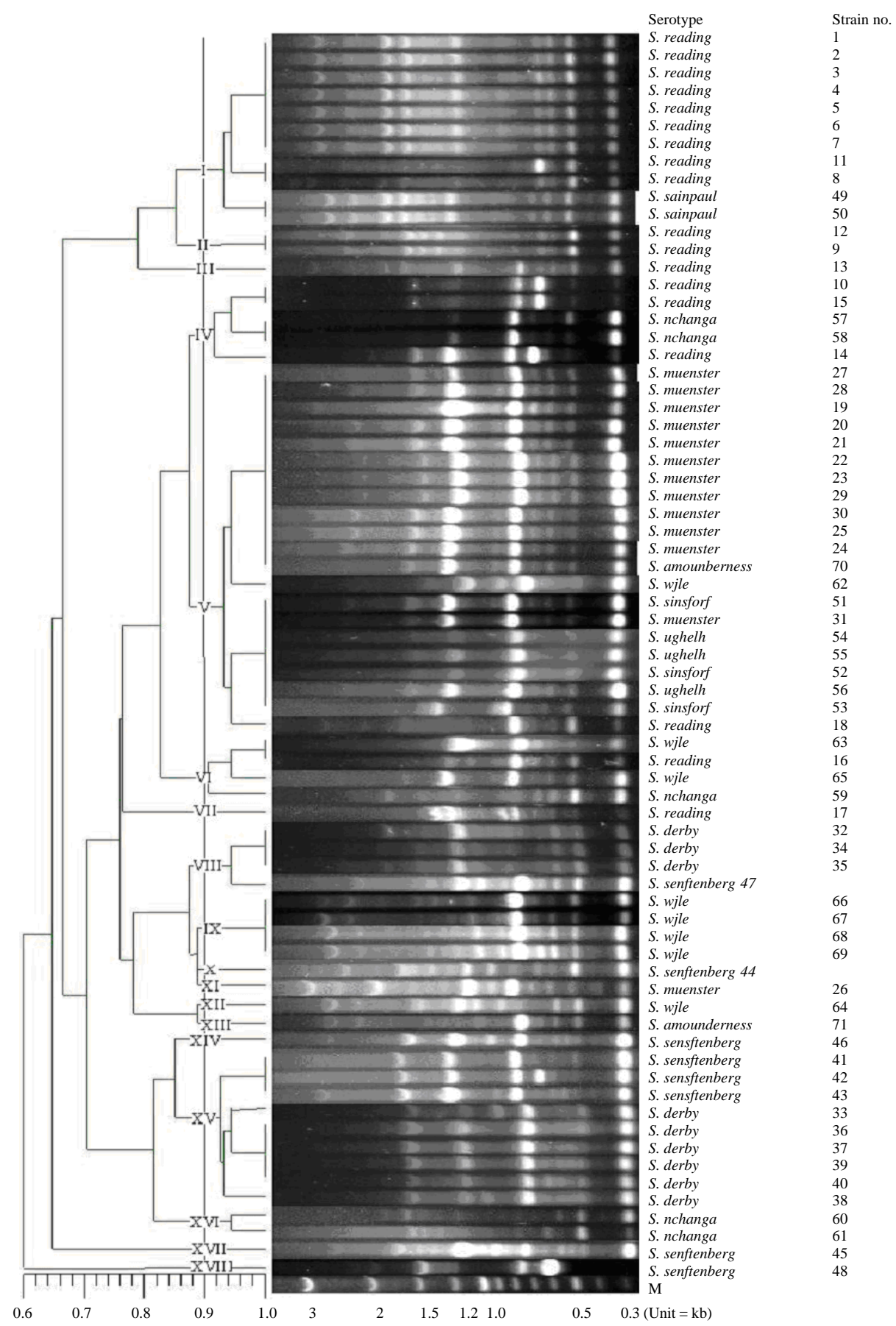

Fig. 1: ERIC-PCR dendrogram exhibiting the genetic relatedness of Salmonella $(n=71)$ isolated from pig carcass. The percentage of similarities among strains was determined using Dice coefficient and the clustering was performed by UPGMA

further explored. The results show that from the same abattoir, the same serotype of Salmonella isolates from carcass surface are of the same genotype or even exactly the same fingerprint. For example, what were isolated from the surface of the carcass CR strains were 1 st- 8 th,
$S$. reading and I genotype and carcass meat samples were Salmonella isolates genotype polymorphism. For example what were isolated from CR 10-13th carcass meat like strains were $S$. reading but the genotypes belonging to IV, I, II, III. Moreover, some were isolated from the same 
abattoir, the carcass of the same serotype of surface and carcass Salmonella isolates from meat samples or fingerprints are exactly the same genotype. For example, what were isolated from the surface of the 1 st -8 th $C R$ carcass and the carcass meat strains kind of 11 strains were $S$. reading and I genotype; isolated from the HS 24 strains of the surface of the carcass and carcass meat samples and 25 strains were $S$. muenster $\mathrm{V}$ genotype and so on. Then from the angle of genes, it can be shown that pigs during the course of slaughter and processing can have cross-contamination among different strains on the carcass surface. The pollution is from the carcass meat samples infected before slaughtered. It also further confirms that the sources of genotype and the same serumtype are relevant.

The outbreak and prevalence of Salmonellosis epidemic have become a global public health problem, so the detection of food-borne Salmonella and the homology analysis are also significantly important. As a rapid and effective molecular typing technique, ERIC-PCR not only can be applied to the genotyping of Salmonella but also can be used in tracing pathogen in food.

\section{CONCLUSION}

This study identifies the relevance between genotypes of the strain of Salmonella and the same serotype and clarifies the pollution source of Salmonella in slaughtered carcass and muscle surface so as to provide scientific basis for the monitoring of Salmonella contamination and the selection of critical control points during the process of pig slaughtering and the prevention and control of food-borne diseases.

\section{ACKNOWLEDGEMENTS}

This research is financially supported by the Agricultural Science and Technology Achievements Transformation Foundation of China (Contract no: 2007GB2C100109), National Science and Technology Support Projects of China (Contract no: 2009BADB9B01), Higher School Science Research Project in Anhui province of China (Contract no: KJ2008B056).

\section{REFERENCES}

Borges, L.G.D.A., V.D. Vechia and G. Corcao, 2003. Characterisation and genetic diversity via REP-PCR of Escherichia coli isolates from polluted waters in southern Brazil. FEMS Microbiol. Ecol., 45: 173-180.
Burr, M.D., K.L. Josephson and I.L. Pepper, 1998. An evaluation of ERIC PCR and AP PCR fingerprinting for discriminating Salmonella serotypes. Lett. Applied Microbiol., 27: 24-30.

Hulton, C.S.J., C.F. Higgins and P.M. Sharp, 1991. ERIC sequences: A novel family of repetitive elements in the genomes of Escherichia coli, Salmonella typhimurium and other enterobacteria. Mol. Microbiol., 5: 825-834.

Kumar, R., P.K. Surendran and N. Thampuran, 2008. Molecular fingerprinting of Salmonella enterica subsp. enterica typhimurium and Salmonella enterica subsp. enterica derby isolated from tropical seafood in South India. J. Mol. Biotechnol., 40: $95-100$.

Li, Y., X.A. Jiao, J.Z. Wei, W. Wang, Q. Wang, C.F. Li and G.J. Wang, 2008. Analysis on the serotypes and drug-susceptibility of Salmnella isolates from pig carcass. Chinese J. Zoonoses, 24: 67-70.

Lim, H., K.H. Lee, C.H. Hong, G.J. Bahk and W.S. Choi, 2005. Comparison of four molecular typing methods for the differentiation of Salmonella spp. Int. J. Food Microbiol., 105: 411-418.

Rasschaert, G., K. Houf, H. Imberechts, K. Grijspeerdt, L. de Zutter and M. Heyndrickx, 2005. Comparison of five repetitive-sequence-based PCR typing methods for molecular discrimination of Salmonella enterica isolates. J. Clin. Microbiol., 43: 3615-3623.

Swanenburg, M., H.A.P. Urlings, D.A. Keuzenkamp and J.M.A. Snijders, 1998. Validation of ERIC PCR as a tool in epidemiologic research of Salmonella in slaughter pigs. J. Ind. Microbiol. Biotechnol., 21: 141-144.

Van Lith, L.A.J.T. and H.J.M. Aarts, 1994. Polymerase chain reaction identification of Salmonella serotypes. Lett. Applied Microbiol., 19: 273-276.

Versalovic, J., T. Koeuth and J.R. Lupski, 1991. Distribution of repetitive DNA sequences in eubacteria and application to fingerprinting of bacterial genomes. Nucleic Acids Res., 19: 6823-6831.

Yu, L., J. Xin-An, W. Jian-Zhong, W. Gui-Jun, L. ChunFen, Z. Ji-Gui and H. Jin-Lin, 2007. Investigation of main microbial indices on pig carcass in Hefei. Chinese J. Health Lab. Teachnol., 17: 1191-1192.

Zhang, H.M., S. Lei and L. Lin, 2007. Fingerprinting genomes of class 1 integron among Salmonella stains by enterobacterial repetitive intergenic consensus PCR. J. Yunnan Agric. Univ., 22: 467-470. 